\title{
Drag forces in statistically stationary and homogeneous submerged granular flows
}

\author{
E. Nucci, ${ }^{*}$ A. Armanini, and M. Larcher ${ }^{\dagger}$ \\ Department of Civil, Environmental and Mechanical Engineering, CUDAM, University of Trento, Trento, Italy
}

(Received 1 August 2018; published 19 April 2019)

\begin{abstract}
The paper deals with the interaction forces between the two phases of a granular flow immersed in water, analyzing in detail the effects on the drag of the concentration of the solid phase and of the interactions between velocities of the two phases and particle concentration. According to the system of equations, the relations between these variables are strongly nonlinear. In particular, this article addresses two issues: how the drag force varies as a function of the concentration only, and whether it is proper to determine the averaged drag force only as a function of the time-averaged values of velocities and concentration. We demonstrate, in fact, that by separating the average and the fluctuating part of each quantity, new terms arise representing a residual drag. To better understand the role of the interphases forces, we performed a series of tests on a two-phase flow (spherical particles in water) through a vertical column in a statistically stationary and homogeneous condition.
\end{abstract}

DOI: 10.1103/PhysRevE.99.042904

\section{INTRODUCTION}

Granular materials are agglomerations of macroscopic particles characterized by peculiar behavior with respect to the three standard states of matter: solid, liquid, and gas. According to Jaeger et al. [1], the granular state "should therefore be considered an additional state of matter in its own right." Due to its unique features, granular matter attracts great interest, with applications in different sectors, e.g., physics, chemistry, pharmaceuticals, agriculture, energy production, industrial engineering, and environmental engineering [2,3].

A first classification of the granular flows is based on the nature of the fluid surrounding the particles (interstitial fluid): if the interstitial fluid is a gas, e.g., air, the particles are considered dry granular flows, while if the interstitial fluid is a liquid, like water, they are treated as submerged granular flows. In more complex cases, the interstitial fluid may be composed simultaneously of air and water, wet granular flows [4], or other liquids.

The differences between these types of flows depend on the interaction between the phases: when the interstitial space between particles is occupied by air, due to the large density difference between the two phases, the interaction forces between the two phases is usually negligible, whereas if it is occupied by water, these forces become important and dependent on the degree of dilution [5].

In this paper, we focus on submerged granular flows driven by gravity, with the ultimate goal being to better understand

\footnotetext{
*elena.nucci@unitn.it

${ }^{\dagger}$ Present address: Faculty of Science and Technology, Free University of Bozen-Bolzano, Bolzano, Italy.
}

Published by the American Physical Society under the terms of the Creative Commons Attribution 4.0 International license. Further distribution of this work must maintain attribution to the author(s) and the published article's title, journal citation, and DOI. the important features of debris flows, which are extraordinary sediment transport phenomena, typical of mountainous environments, able to move huge amounts of solid material in a very short time [6,7]. The recent urbanization of these areas has increased the debris flow risk, also causing loss of lives and property damage. For these reasons, a deeper knowledge of its mechanical behavior is as important as it is urgent.

In numerical modeling, debris flows are often treated as homogeneous fluids (mixtures of sediment and interstitial fluid), i.e., by adding together the equations of the two phases [8]. In this case, interaction forces do not appear, since they cancel each other, as will be better explained in Sec. II.

Regarding the two-phase models of debris flows, instead there are different criteria in the literature to express the forces of interaction among the solid particles [6-12], while the modeling of the interaction forces between the solid and liquid phases is still lacking in a convincing approach from a physical point of view.

The objective of the paper is to analyze this problem, first by using a theoretical approach based on the balance equations that govern granular flows, and then through a series of laboratory experiments of the granular flow driven by water in a vertical column.

The paper is organized as follows: Section I covers some fundamentals of the mechanics of granular flows, the twophase approach, and the rheological relations. In Sec. II we analyze in detail the interaction forces between the liquid and the granular phases, and we broaden the definition of drag on a group of noncohesive particles. We derive a relation for the drag force that is valid also in the case of high solid concentrations. Section III describes the experimental setup and the measurement techniques. In Sec. IV we explain the averaging procedure, and we derive the time-averaged equations of the flow. Section V describes the results of the experimental investigation. In Sec. VI we present some problems due to the fluctuations in velocities on nonlinear features of the drag force. Section VII contains the conclusions. 


\section{A. Flow equations}

The mechanics of submerged granular flows is defined by the equations of mass and momentum conservation of the two phases $[9,13]$ in an infinitesimal control volume, but sufficiently large compared to the particle dimensions. The system, written separately for each phase, is

$$
\begin{aligned}
\frac{\partial \rho^{\beta}}{\partial t}+\frac{\partial\left(\rho^{\beta} u_{i}^{\beta}\right)}{\partial x_{i}} & =0 \\
\frac{\partial\left(\rho^{\beta} u_{i}^{\beta}\right)}{\partial t}+\frac{\partial\left(\rho^{\beta} u_{i}^{\beta} u_{j}^{\beta}\right)}{\partial x_{j}} & =\rho^{\beta} G_{i}^{\beta}+\frac{\partial \sigma_{i j}^{\beta}}{\partial x_{j}}+F_{i}^{\beta}
\end{aligned}
$$

with $\beta=g$ for the granular phase and $\beta=f$ for the interstitial fluid; $\rho^{g}=c \rho_{s}$ is the density of the granular phase, and $\rho^{f}=$ $(1-c) \rho_{w}$ is the density of the liquid phase; $c$ is the volume concentration of the solid phase; $\rho_{s}$ is the material density of the grains; and $\rho_{w}$ is the water density. For each phase, $u_{i}^{\beta}$ is the generic component of the velocity vector, $\sigma_{i j}^{\beta}$ is the generic component of the tensor of the internal stresses, and $F_{i}^{\beta}$ is the component of the interaction force (per unit volume). $G_{i}^{\beta}$ represents the body forces acting on each constituent in the $i$ th direction. In the present case, we assume $G_{i}^{\beta}=-g \partial z / \partial x_{i}$, where $z$ represents the vertical rising direction and $g$ is the acceleration of gravity.

To solve the system (1), closure relations for the stresses of the liquid phase $\sigma_{i j}^{f}$, for the stresses of the granular phase $\sigma_{i j}^{g}$, and a relation for the interaction forces $F_{i}^{g}$ are needed.

\section{B. Flow regimes}

If the interstitial fluid is water, the related internal stresses are well described by the Newtonian rheology. The rheology of the granular phase is more complex and still presents some unresolved issues. Generally, the mechanics of the grain-grain interaction depends on the contacts between the particles, and two different types of interactions usually occur: almost instantaneous and binary contacts or long-lasting contacts involving more particles at the same time. A distinction between these two conditions is based on the characteristic time scales of the types of interaction $[14,15]$.

When the contacts are instantaneous, the granular flow is characterized by continuous, partially elastic collisions between the particles. In this regime, the rheology has been described, since the 1970s, by applying a kinetic theory derived in analogy with the kinetic theory of gases, in which the thermodynamic temperature is replaced by the granular temperature, which plays a similar role in generating pressure and governing the mass, momentum, and energy transports $[2,16,17]$.

With reference to a system composed of $N_{p}$ particles in a control volume (see Fig. 1), we define the average velocity of the granular phase as

$$
u_{i}^{g}=\frac{\sum_{k=1}^{N_{p}}\left(u_{i}^{p}\right)_{k}}{N_{p}},
$$

where $i$, according to the indicial notation, represents the index of the generic directions of the flow, $\left(u_{i}^{p}\right)_{k}$ is the $i$ th component of the velocity of each single particle, and $N_{p}$ is the

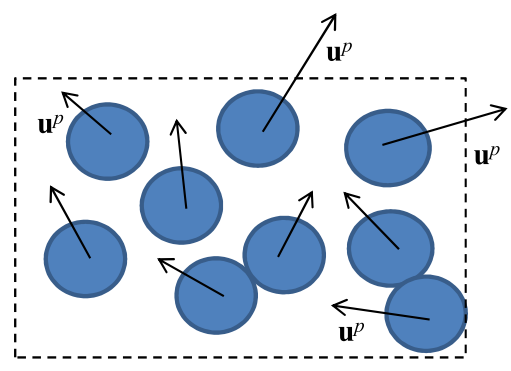

FIG. 1. Representation of the vectors of the velocities $\mathbf{u}^{p}$ of the $N_{p}$ particles inside a control volume.

number of particles inside the control volume. In this respect, the granular temperature is defined as

$$
T=\frac{1}{3} \sum_{i=1}^{3} \frac{\sum_{k=1}^{N_{p}}\left[\left(u_{i}^{p}\right)_{k}-u_{i}^{g}\right]^{2}}{N_{p}} .
$$

The granular temperature changes in space and in time, and also represents the kinetic energy, per unit mass, of the fluctuating particles.

This procedure should be valid only if the control volume is infinitesimal and if the number of particles inside it is very large. Nonetheless, in most applications of the kinetic theory to granular flows (dense gas analogy), this process is considered valid even in the presence of particles with finite dimensions by assuming that all the particle's properties are concentrated in its center [2,6,18,19].

On the other hand, when the contacts are long-lasting and involve more particles at the same time, the gas analogy becomes problematic. In the application to channel flows, this condition usually occurs near the bottom of the flow: if the bottom is a rigid surface, the grain-wall interaction leads to a slip velocity and to a finite granular temperature at the wall, and the flow is considered to be in the dense regime [19]. In contrast, if the bottom is represented by a loose packing bed of the same material as the granular flow, the velocity of the granular phase tends asymptotically to zero while approaching the mobile bed. In this limit, the granular temperature also vanishes, but the internal shear stress of the granular phase is nonzero [5]. This type of flow is referred to as the frictional regime. In a channel flow of granular material on a mobile bed driven by gravity, the collisional and the frictional regimes coexist across the entire flow through an intermittent mechanism in space and time [20]: the collisional regime governs the region near the free surface, and the frictional regime governs the layer approaching the mobile bed. However, even in this layer, the instantaneous contacts are not entirely absent, although they occur with less and less frequency as one moves toward the immobile bed. They (and consequently the granular temperature) exert an important role in the process of energy dissipation [5,21].

The relative weights of the three terms on the right-hand side of the second equation of system (1) depend on the concentration: in the dilute case, the internal stresses of the solid phase are negligible compared to the phase-interaction forces, whereas while the solid concentration increases, the influence of the internal stresses of the granular phase increases until the interaction forces become negligible. When the concentration 
tends to its maximum value (random packing concentration), in the momentum equation also the internal stresses of the liquid phase are negligible [5].

\section{INTERPHASES FORCES}

In the momentum balances, the term $F_{i}^{\beta}$ represents the coupling force between the two phases. Since due to the principle of reciprocity, $F_{i}^{g}=-F_{i}^{f}$, by adding together the momentum equations of the liquid and the solid phases, the interaction forces cancel each other so that they do not appear in the momentum balance equation of the mixture.

The interactions between the two phases are ascribed to different causes:

(i) The stress gradients of the liquid phase (buoyancy effect).

(ii) The relative velocity between the two phases (drag effect).

(iii) The relative acceleration between particles and fluid (added mass).

(iv) The unsteady diffusion of swirling in the boundary layer around the particles, which is a history force (Basset force).

In the first instance, in statistically stationary channel flow, the added mass and Basset force are usually neglected $[2,5,22-25]$, and the interaction force is

$$
F_{i}^{f}=c\left(\frac{\partial p^{f}}{\partial x_{i}}-\frac{\partial \tau_{i j}^{f}}{\partial x_{j}}\right)-D_{i},
$$

where the first two terms on the right-hand side represent the buoyancy, and the third term is the drag force.

\section{A. The drag force of a noncohesive population of particles}

There are many examples of granular flows where drag plays an important role, e.g., sedimentation, fluidization, flows in a porous medium, etc. [26]. In general, the expression for the drag, $D_{i}$ in Eq. (4), on the agglomeration of particles is obtained from the expression for the drag $F_{D}$ exerted by a uniform flow, characterized by an undisturbed flow velocity $U_{o}$, surrounding an isolated particle:

$$
F_{D}=\frac{\pi d^{2}}{4} \rho_{w} C_{D} \frac{U_{o}^{2}}{2}
$$

where $C_{D}$ is the drag coefficient, which is a function of the particle's Reynolds number $\left(\operatorname{Re}_{p}=U_{o} d / v_{w}\right)$, where $v_{w}$ is the kinematic viscosity of the liquid phase, in this case water, and of the shape of the particles.

Although many expressions for the drag coefficient exist in the literature, in this framework we assume the relation of Dallavalle [27], which is valid for spherical particles over a large range of Reynolds numbers:

$$
C_{D}=\left(\frac{4.8}{\sqrt{\mathrm{Re}_{p}}}+0.63\right)^{2} .
$$

We derived the drag of the group of particles, $D_{i}$, by substituting the velocity $U_{o}$ in Eq. (5) with the relative velocities between the two phases, $\left(u_{i}^{f}-u_{i}^{g}\right)$, by multiplying Eq. (5) by the number of particles $n$ per unit volume, and by introducing a suitable function $f^{p}$ of the concentration, accounting for the influence of the other particles on the flow field. The number density of particles within a control volume is related to the volumetric concentration $c$ and to the particle size $d$, i.e., $n=6 c / \pi d^{3}$. Finally, we have

$$
D_{i}=\frac{3}{4} \frac{\rho_{w}}{d} c C_{D}\left(u_{i}^{f}-u_{i}^{g}\right)^{2} f^{p}
$$

In principle, if velocities and concentration are statistically stationary functions, we expect that by averaging over time Eq. (7) (a nonlinear equation) some residual terms arise due to possible correlations between the fluctuating components of $u_{i}^{f}, u_{i}^{g}$, and $c$. These correlations cannot be excluded a priori. This point will be discussed in detail in Sec. VI.

\section{B. Particle population drag function $f^{p}$}

There are several methods in the literature to take into account the effect of the concentration of a group of particles on the drag coefficient of an individual particle. For example, in fluvial hydraulics the presence of particles is considered by modifying the von Kármán constant, or by introducing some parameters accounting for the turbulence damping produced by a cluster of particles [28]; in fluidized beds, a voidage function (or correction function) is often introduced, defined as the ratio between the drag force on a particle when taking into consideration the effect of neighboring particles, and the drag force exerted on the same particle when falling isolated in still water $[29,30]$, or the ratio between the drag force on a particle in a fluid-multiparticle interaction system and the drag force on an unhindered particle, subject to the same volumetric flux of fluid [31]. Since in this paper we adopted a fully two-phase approach with respect to a noncohesive population of particles inside the control volume, we introduced the population drag function $f^{p}$, defined as the ratio between the modulus of the drag force $D_{i}$ of the group of $n$ particles and $n F_{D}$, where $F_{D}$ is the drag force experienced by an isolated particle under the same flow velocity, i.e.,

$$
f^{p}=\frac{\left|D_{i}\right| \pi d^{3}}{F_{D} c 6}
$$

The population drag function $f^{p}$ should tend to 1 when the concentration tends to 0 , and increase as the concentration increases.

Moreover, in the limit condition in which the timeaveraged concentration tends to its maximum value $c_{\mathrm{rp}}$ (random-packing concentration) $\left(c \rightarrow c_{\mathrm{rp}}\right)$, the motion of the interstitial fluid relative to the granular matrix corresponds to a filtration flow through the granular matrix, so we expect that the drag corresponds to that of the flow in a porous medium.

With reference to the notation in Fig. 2, according to Darcy's law the velocity $u_{D}$ (Darcy velocity) is the ratio between the flow rate $Q$ and the total cross-sectional area $A$. By definition, the Darcy velocity is a fictitious velocity calculated as if the flow would occur across the entire cross section of the sediment sample $A$. The flow actually takes place only through interconnected pore channels, $A_{v}$, and the ratio between the flow rate and $A_{v}$ is the seepage velocity $\left(u_{v}^{f}\right)_{i}$.

The velocity of the fluid phase of system 1 corresponds to the average of the seepage velocities inside the control 


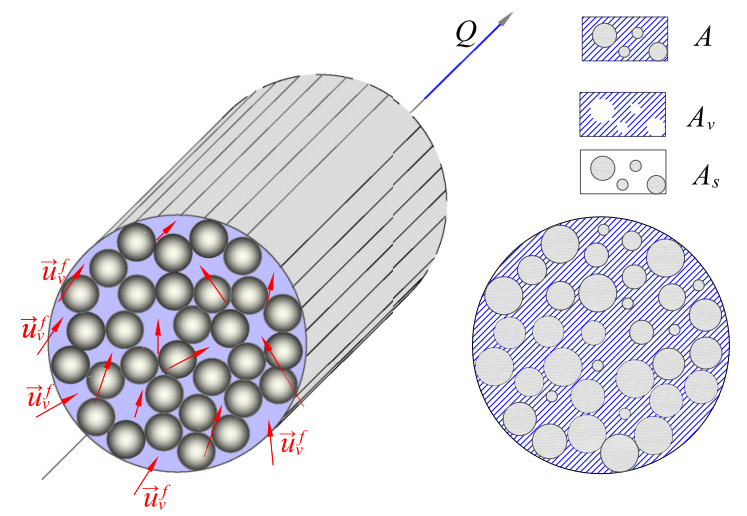

FIG. 2. A cross section $A$ of the granular flow sample. $Q$ is the flow rate across $A$, with a fictitious velocity $u_{D}$. The velocity of the fluid phase is represented by the velocity in the interstices $\left(u_{v}^{f}\right)_{i} . A_{v}$ is the area occupied by the fluid, while $A_{s}$ is the area occupied by the particles.

volume,

$$
u_{i}^{f}=\frac{1}{V_{v}} \int_{V_{v}}\left(u_{v}^{f}\right)_{i} d V_{v},
$$

where $V_{v}$ is the volume of the voids in the control volume. The relation between the Darcy velocity and the average seepage velocity, i.e., the velocity of the liquid phase in system (1), is

$$
\left(u_{D}\right)_{i}=u_{i}^{f}(1-c) .
$$

The relation between $\left(u_{D}\right)_{i}$ and the hydraulic gradient $\partial h^{*} / \partial x_{i}$ depends on the hydraulic conductivity $K_{i}$ (Darcy's equation [32]). If the Reynolds number relevant to $\left(u_{D}\right)_{i}$ is small enough, i.e., in the laminar regime, this relation is linear:

$$
\left(u_{D}\right)_{i}=K \frac{\partial h^{*}}{\partial x_{i}},
$$

where $h^{*}$ is the hydraulic head and, since the medium is isotropic and homogeneous, the hydraulic conductivity is assumed to be a scalar. $K$, which dimensionally is a velocity, is defined as the specific discharge per unit of hydraulic gradient. It is usually expressed as $K=\mathrm{kg} / \mathrm{v}_{w}$, including the influences of the fluid properties and of the solid matrix. $k$ represents the intrinsic permeability, which has the dimension of surface area [33,34]. According to Carman [35], in the laminar regime of flows in porous media, the permeability is $k=(1-c)^{3} c^{-2} d^{2} 180^{-1}$.

In uniform flow conditions, when $c \rightarrow c_{\mathrm{rp}}$, the momentum equation of the liquid phase of system (1) reduces to the balance between the drag force and the weight of the liquid phase, i.e.,

$$
\frac{4}{3} \frac{\rho_{w}}{d} c C_{D}\left(u_{i}^{f}-u_{i}^{g}\right)^{2} f^{p}=-\rho_{w} g(1-c) \frac{\partial z}{\partial x_{i}} .
$$

From Eq. (12), an analytical expression of the population drag function $f_{\mathrm{rp}}^{p}$, valid at random-packing concentration and in the laminar regime, can be derived:

$$
f_{\mathrm{rp}}^{p}=10 \frac{c_{\mathrm{rp}}}{\left(1-c_{\mathrm{rp}}\right)} .
$$

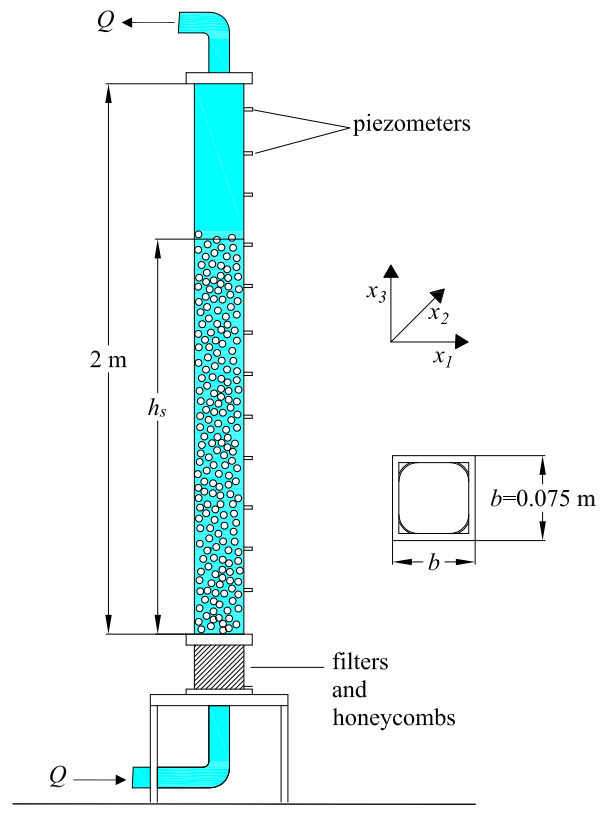

FIG. 3. Sketch of the column setup used for the experiments.

This relation was proposed earlier by several authors $[33,34,36]$. As we explain better in the next sections, we measured the values of the drag of the group of particles for different values of the volume concentration, and by comparing these values with the values of the drag of the single sphere, we obtained the values of the population drag function for different values of the concentration. Finally, in Sec. VI we propose an expression for the population drag function valid for $0 \leqslant c \leqslant c_{\mathrm{rp}}$.

\section{EXPERIMENTAL INVESTIGATIONS}

The experimental investigation was carried out in the Hydraulics Laboratory of the University of Trento. The experimental setup was constituted by a 2-m-long vertical duct hereafter called the column, with an almost square section (Fig. 3), fed from the bottom with an adjustable water discharge measured with an electromagnetic flow meter with an error of the full scale of $1 \%$. A system of filters and honeycombs was inserted at the entrance of the column in order to guarantee the uniformity of the flow. Before each test the column was filled, in the absence of water, by a known volume of solid noncohesive spheres, with constant diameter $d=6 \mathrm{~mm}$ and density $\rho_{s}=2250 \mathrm{~kg} / \mathrm{m}^{3}$. The measured value of the random-packing concentration of these particles is $c_{\text {rp }}=0.67$. When the liquid discharge was injected from the bottom, the solid particles, due to the drag force, expanded, reaching in a short time a statistically stationary configuration. This arrangement, similar to that of previous research on fluidized beds [31,37-39], was such that the ratio between the diameter $D$ of the circle inscribed in the section of the column and the diameter of the particles was less than 25 in order to avoid any possible $2 \mathrm{D}$ instability, and larger than 10 in order to avoid the particle arching effect within the tube [37]. With respect to the phenomenon of the voidage wave instability, according to the analysis proposed in [39], all the 
tests were performed with combinations of specific flow rates and concentrations (expansion curve of the fluidized bed), where the wavy instability no longer occurred.

Finally, in order to guarantee the stationarity and homogeneity of the flow, the measurements were initiated after a period sufficiently long to ensure a constant water discharge $(\sim 15 \mathrm{~min})$.

\section{A. The measurement of the velocity and of the granular temperature}

The instantaneous velocities of the particles were measured by means of two synchronized cameras with acquisition frequencies between 60 and $500 \mathrm{fps}$. We set the two cameras one above the other in order to record simultaneously two contiguous regions of the flow. Then we moved the two cameras rigidly in the vertical direction and recorded as many times as needed to acquire data from the entire measuring section (usually three times). Due to the logistics of the laboratory, we could not perform optical measurements (local velocities, granular temperature, and concentration) in the first $30 \mathrm{~cm}$ of the column.

To measure the velocity of each single particle visible through the transparent wall, we adopted an imaging method based on the Voronoï polygons to process the images taken by the high-speed camera. The interested reader can refer to $[10,40]$ for a full description of the method. For every frame, the particles' positions were recorded and the particles on one frame and the next were then paired based on the goodness-of-match between the shapes of their Voronoï cells. The velocities were finally derived from the corresponding displacements (optical method).

The velocity of the fluid phase was measured by injecting into the flow small and light tracking particles $\left(d_{t}=0.6 \mathrm{~mm}\right.$ and $\rho_{t}=1050 \mathrm{~kg} / \mathrm{m}^{3}$ ) by assuming that the velocity of these particles represented the velocity of the liquid phase and by measuring their velocities with the optical method.

\section{B. The measurement of the pressure}

The water pressure in the column was measured by means of a battery of piezometers spaced $15 \mathrm{~cm}$ apart along the column (see Fig. 3). Figure 4 shows the typical distribution of the pressure of the fluid phase. These devices sensibly dump the possible pressure oscillations, and the accuracy of the head measure is about $1-2 \mathrm{~mm}$.

It is possible to appreciate two different behaviors of the pressure gradient (Fig. 4). A considerably linear distribution of the pressure, and an even more remarkable uniform concentration distribution, as it is explained in Sec. V [Fig. 7(c)], characterize the two-phase region. In this region, the pressure gradient is likely determined by the drag force between the water and the particles. An external region, identified by the absence of particles, was characterized by a pressure gradient, also linear, but with a definitely lesser slope, induced by the wall resistance of the duct only. The transition between the two regions is sharp. All the other tests had similar behaviors.

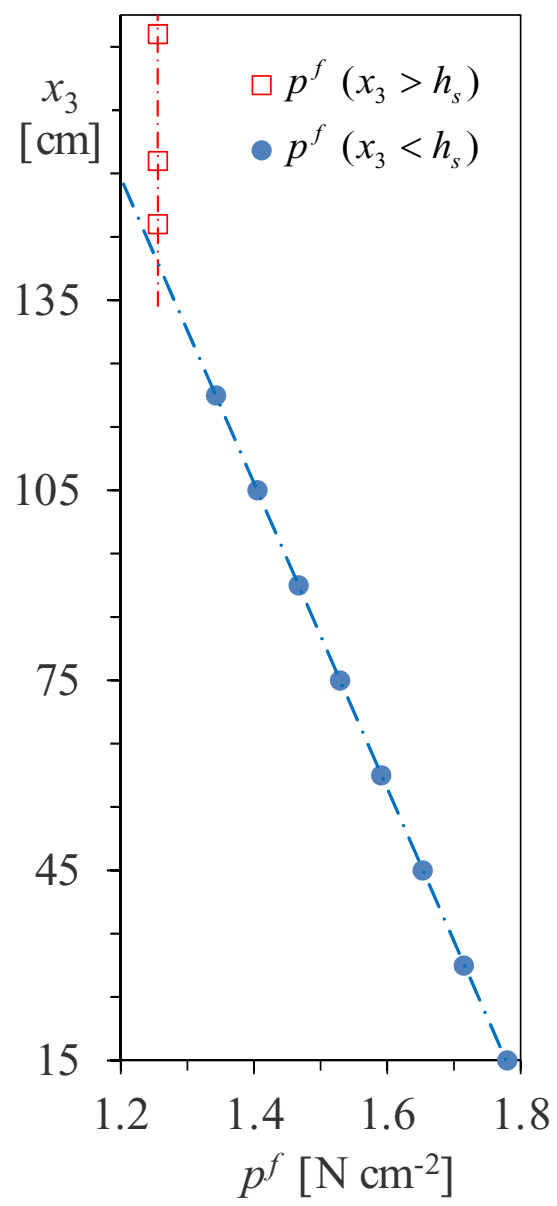

FIG. 4. Distribution of the fluid pressure along the column. The dotted blue horizontal line identifies the height of the two-phase flow region. $Q=80 \mathrm{~L} / \mathrm{min}$.

\section{The measurement of the concentration}

The local particle concentration was measured by means of the optical method adopted for recording the particles' velocity. In particular, we identified an area inside the record where we defined the $2 D$ particle concentration $\lambda_{a}$ based on the fact that in a system of identical, regularly organized spherical particles the hypothetical disposition of the particles is such that the three particles belong to a plane parallel to the transparent wall. According to this disposition of the particles, the $2 D$ particle concentration $\lambda_{a}$ is defined by the ratio between the area of the triangle connecting the centers of three particles and the net cross section between this triangle and the three particles.

By adopting a procedure similar to that for the linear concentration [41], we derived a relation between the volume concentration $c$ and the areal concentration $\lambda_{a}$ for spherical particles:

$$
\frac{c}{c^{*}}=\left(\frac{\lambda_{a}}{\lambda_{a^{*}}}\right)^{3 / 2},
$$

where $c^{*}$ and $\lambda_{a^{*}}$ are the theoretical maximum values of the volume and of the superficial concentration, respectively, i.e., for spherical particles $c^{*}=\pi / 3 \sqrt{2} \sim 0.74$ and $\lambda_{a^{*}}=$ $\pi / 2 \sqrt{3} \sim 0.90$. 


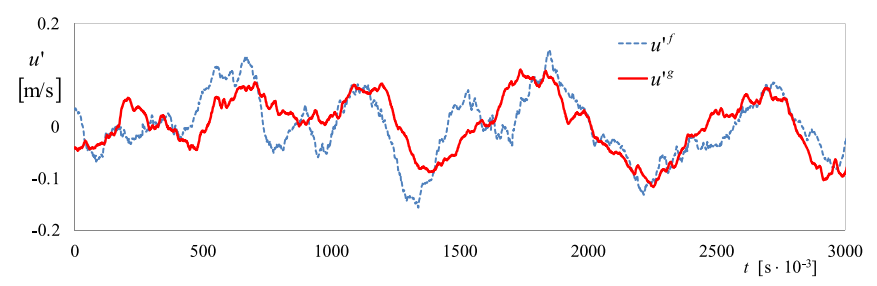

FIG. 5. Time fluctuations of velocities of the liquid and of the solid phases $(Q=67 \mathrm{~L} / \mathrm{min}$ and $C=0.3$ ).

We measured also the average volume concentration $C$ for each test by dividing the volume of the two-phase region by the total volume of the particles contained in it (volumetric method). The goodness of the measuring methods adopted was checked by comparing the average local concentration with the average of the volume concentration $C$, as we will show in Sec. V.

\section{DERIVATION OF THE AVERAGING EQUATIONS OF THE FLOW}

The measurements of the velocities of the solid and liquid phases, defined according to Eq. (2), show that these quantities strongly fluctuate in time. To put in evidence a possible correlation between the two velocities, we filtered the highfrequency component of the signal of the water velocity.

From Fig. 5, which presents a specimen of the fluctuations, it is possible to appreciate the good correlation between these two signals.

This observation leads us to hypothesize that these large fluctuations are induced by wall turbulence, rather than by the agitation of the particles at the scale of the collisions between the grains. To validate this hypothesis, we did some tests by measuring the statistics of turbulence of a flow in the column in the absence of particles and comparing these with the same statistics for the same flow rate of water.

In this regard, it is useful to point out that in two-phase fluid mechanics, two types of averaged variables exist: the phase average and the mass weighted average. We decided to adopt the first type, consistent with the definition of the flow of the granular phase at the scale of particle collisions. On the other hand, Hsu et al. [42,43] and Greimann et al. [44] based the definition of the Reynolds scaling fluctuations on the mass weighted average.

In other words, we defined the averaged values of a generic fluctuating property $\psi$ of the flow as

$$
\bar{\psi}=\frac{1}{T_{m}} \int_{T_{m}} \psi d t,
$$

where $T_{m}$ is a period sufficiently long to consider the flow statistically stationary, and we derived the fluctuating component of $\psi$ :

$$
\psi^{\prime}=\psi-\bar{\psi}
$$

By definition, the average of the fluctuating term is null: $\overline{\psi^{\prime}}=0$.

Table I shows the results of this procedure. The table also shows that the presence of the solid phase slightly alters the turbulence intensity of the liquid phase.
TABLE I. Comparison of the average velocities and of the turbulent statistics of the fluid phase in the presence and in the absence of particles.

\begin{tabular}{lccc}
\hline \hline & & Clear water flow & Two-phase flow \\
\hline$\overline{\overline{u_{3}^{f}}}$ & $(\mathrm{~m} / \mathrm{s})$ & 0.23 & 0.22 \\
$\overline{u_{3}^{\prime f} u_{3}^{\prime f}}$ & $\left(\mathrm{~m}^{2} / \mathrm{s}^{2}\right)$ & 0.021 & 0.022 \\
$\overline{u_{1}^{\prime f} u_{1}^{\prime f}}$ & $\left(\mathrm{~m}^{2} / \mathrm{s}^{2}\right)$ & 0.001 & 0.001 \\
\hline \hline
\end{tabular}

On the basis of these considerations, therefore, it seems plausible to adopt also in our case the scale separation of the solid phase, already introduced by Greimann et al. [44] and by Hsu et al. $[42,43]$ in the analysis of sediment transport in a channel made with kinetic theories of granular flows.

It is then appropriate to average the flow equations [system (1)] over a time at the scale of the turbulence fluctuations in the liquid phase (Reynolds scale). The flow equations are then obtained by applying the Reynolds decomposition to the equations of system (1) and averaging them over time:

$$
\begin{aligned}
& \frac{\partial}{\partial t}(1-\bar{c})+\frac{\partial}{\partial x_{i}}\left((1-\bar{c}) \overline{u_{i}^{\prime f}}+\overline{c^{\prime} u_{i}^{\prime f}}\right)=0, \\
& \frac{\partial}{\partial t}\left((1-\bar{c}) \overline{u_{i}^{\prime f}}+\overline{c^{\prime} u_{i}^{\prime f}}\right)+\frac{\partial}{\partial x_{j}}\left((1-\bar{c})\left(\overline{u_{i}^{\prime f} u_{j}^{f}}+\overline{u_{i}^{\prime f} u_{i}^{\prime f}}\right)\right. \\
& \left.+\overline{u_{i}^{f}} \overline{c^{\prime} u_{i}^{\prime f}}+\overline{u_{j}^{f}} \overline{c^{\prime} u_{i}^{\prime f}}+\overline{c^{\prime} u_{i}^{\prime f} u_{i}^{\prime f}}\right) \\
& =\bar{c} g \frac{\partial z}{\partial x_{i}}+\frac{1}{\rho_{w}}\left(-\frac{\partial p^{f}}{\partial x_{i}}+\frac{\partial \overline{\tau_{i j}^{f}}}{\partial x_{j}}\right)+\frac{\overline{F_{i}^{f}}}{\rho_{w}}, \\
& \frac{\partial \bar{c}}{\partial t}+\frac{\partial}{\partial x_{i}}\left(\bar{c} \overline{u_{i}^{g}}+\rho_{s} \overline{c^{\prime} u_{i}^{\prime g}}\right)=0, \\
& \frac{\partial}{\partial t}\left(\bar{c} \overline{u_{i}^{g}}+\overline{c^{\prime} u_{i}^{\prime g}}\right)+\frac{\partial}{\partial x_{j}}\left(\bar{c}\left(\overline{u_{i}^{g} u_{j}^{g}}+\overline{u_{i}^{\prime g} u_{j}^{\prime g}}\right)+\overline{u_{i}^{g}} \overline{c^{\prime} u_{j}^{\prime g}}+\overline{u_{j}^{g}} \overline{c^{\prime} u_{i}^{\prime g}}\right) \\
& =\bar{c} g \frac{\partial z}{\partial x_{i}}+\frac{1}{\rho_{s}}\left(-\frac{\partial p^{g}}{\partial x_{i}}+\frac{\partial \overline{\tau_{i j}^{g}}}{\partial x_{j}}\right)+\frac{\overline{F_{i}^{g}}}{\rho_{s}},
\end{aligned}
$$

where $x_{1}$ and $x_{2}$ are the horizontal axes and $x_{3}$ is the vertical rising direction, such that the gravity acceleration is $g=$ $(0,0,-g)$.

\section{RESULTS}

We performed 12 complete tests, with different fluid discharges, as summarized in Table II.

In each test we initially measured the height, $h_{s}$, of the two-phase core region and we derived the volume of the concentration $C$, as explained in Sec. IIIC. Figures 6(a) and 6(b) display the variation of $h_{s} / h_{s o}$ (the extension of the core region divided by its initial value) and $C$ as functions of the dimensionless liquid flow rate $Q / A /(1-C) / \sqrt{g d}=$ $\overline{u_{3}^{f}} / \sqrt{g d}$. As expected, at increasing liquid discharges $Q, h_{s}$ increases while $C$ decreases.

In addition, in each test we measured the distribution of the velocities of the granular and the fluid phases, of the granular 
TABLE II. List of the tests performed.

\begin{tabular}{lcccccc}
\hline \hline Test & 1 & 2 & 3 & 4 & 5 & 6 \\
\hline$Q\left(L \mathrm{~min}^{-1}\right)$ & 12.5 & 20 & 24 & 33 & 45 & 50 \\
$h_{s}(\mathrm{~cm})$ & 30 & 61 & 73 & 81 & 92 & 108 \\
$C$ & 0.67 & 0.64 & 0.62 & 0.59 & 0.48 & 0.44 \\
Test & 7 & 8 & 9 & 10 & 11 & 12 \\
$Q\left(L \min ^{-1}\right)$ & 60 & 67 & 72 & 75 & 80 & 100 \\
$h_{s}(\mathrm{~cm})$ & 132 & 140 & 167 & 184 & 190 & 230 \\
$C$ & 0.36 & 0.30 & 0.27 & 0.24 & 0.20 & 0.05 \\
\hline \hline
\end{tabular}

temperature, of the concentration, and of the pressure of the fluid phase across the core region $h_{s}$.

Figures 7(a), 7(b), and 7(c) show the time-averaged data of two different tests corresponding, respectively, to $Q_{1}=$ $60 \mathrm{~L} / \mathrm{min}$ and $Q_{2}=75 \mathrm{~L} / \mathrm{min}$.

Figure 7(a) presents the vertical distributions of the timeaveraged dimensionless granular velocity $\overline{u_{3}^{g}} / \sqrt{g d}$. The figure shows that this velocity is uniform across the entire two-phase core depth, with values near zero, which can be reasonably considered null. This result is confirmed by the continuity equation of the granular phase if the flow is considered stationary and uniform.

Figure 7(b) shows the vertical distributions of the timeaveraged dimensionless granular temperatures $\bar{T} /(g d)$. The figure confirms that also the distribution of the temperature is constant along the depth. Also in this case, we can consider the gradient as zero.

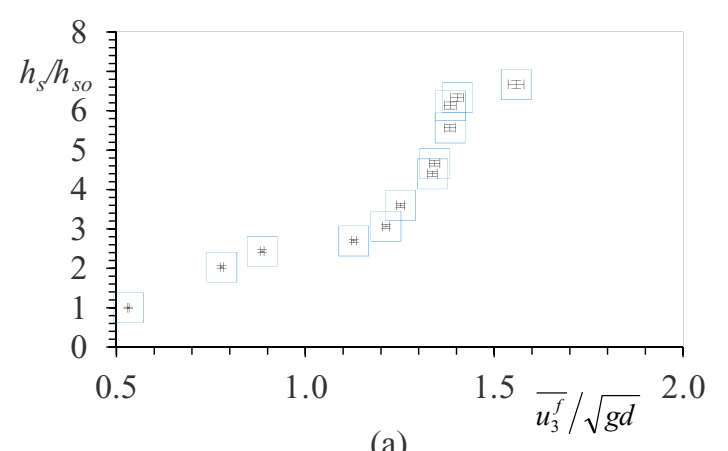

(a)

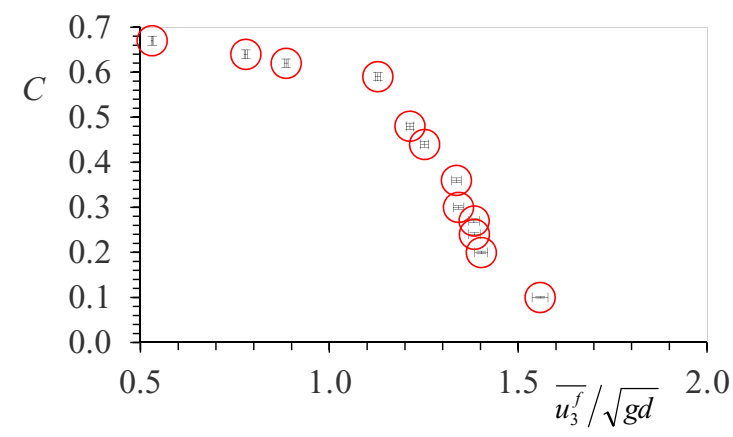

(b)

FIG. 6. (a) Dimensionless values of the measured height of the core region $h_{s} / h_{s o}$ vs $\overline{u_{3}^{f}} / \sqrt{g d}$; (b) measured values of average particle concentration $c$ vs $\overline{u_{3}^{f}} / \sqrt{g d}$.
Finally, Fig. 7(c) presents the vertical distribution of the time-averaged solid concentration and shows that also this variable is reasonably constant along the flow direction. Figure 7(c) shows also the comparisons between the distribution of the average concentration measured with the optical method (see Sec. IIIC) and the value $C$ obtained by the volumetric method. It is possible to appreciate how the two methods give very similar values.

We observed that in all the tests the averages were independent of the time $T_{m}$ for $T_{m}>2 \mathrm{~s}$. For this reason we performed tests with a duration of 6 or $12 s$, depending on the frequency of acquisition.

From the experimental results it emerges that the flow can be reasonably considered to be statistically stationary and homogeneous in the $x_{3}$ direction. These assumptions permit simplifying the time-averaged equations (17) by assuming the following:

(i) The average velocity of the granular phase is null.

(ii) The average velocity of the fluid phase is constant.

(iii) The closure relations for the stresses of the granular phase, based on the kinetic theory, give $\overline{\tau_{i j}^{g}} \propto \partial \overline{u_{i}^{g}} / \partial x_{j}$ and $\overline{p^{g}} \propto \sqrt{\bar{T}}$, and since the vertical gradient of the average velocity of the granular phase is null, the corresponding terms in Eq. (17) are negligible.

(iv) The gradients of the averaged granular temperature and of the averaged concentration are zero, so the gradient of the granular pressure is assumed to be zero.

(v) All the gradients of the correlations of the fluctuating terms can be neglected compared to the gradient of the liquid pressure.

According to the above assumptions, the system (17) reduces to

$$
\begin{gathered}
0=(1-\bar{c})\left(-\rho_{w} g-\frac{\partial \overline{p^{f}}}{\partial x_{3}}+\frac{\overline{\tau_{1,3}^{f}}}{R_{h}}\right)-\overline{D_{3}}, \\
0=\bar{c}\left(-\rho_{s} g-\frac{\partial \overline{p^{f}}}{\partial x_{3}}+\frac{\overline{\tau_{1,3}^{f}}}{R_{h}}\right)+\overline{D_{3}},
\end{gathered}
$$

where $\overline{\tau_{1,3}^{f}}$ represents the shear stresses in the vertical direction on the wall of the column, and $R_{h}$ is the hydraulic radius, i.e., the ratio between the cross-sectional area of the column over the wetted perimeter.

Since we measured the distribution of $\bar{c}$ and of $\partial \overline{p^{f}} / \partial x_{3}$ in each test, we can obtain $\overline{\tau_{1,3}^{f}} / R_{h}$ and $\overline{D_{3}}$ by solving the system (18) and (19). The ratio between $\overline{D_{3}}$ and $F_{D}$ provides the population drag function $f^{p}$ as a function of the volume concentration $\bar{c}$. Figure 8 confirms that the drag increases when the concentration increases. In the same figure, we have also reported the following data fitting curve:

$$
f^{p}=\frac{f_{\mathrm{rp}}^{p} c_{\mathrm{rp}}}{f_{\mathrm{rp}}^{p} c_{\mathrm{rp}}-f_{\mathrm{rp}}^{p} \bar{c}+\bar{c}} .
$$

\section{FURTHER CONSIDERATIONS ON THE DRAG FORCE}

According to the time-averaging procedure adopted, the instantaneous value of the drag force $D_{i}$ has been decomposed 


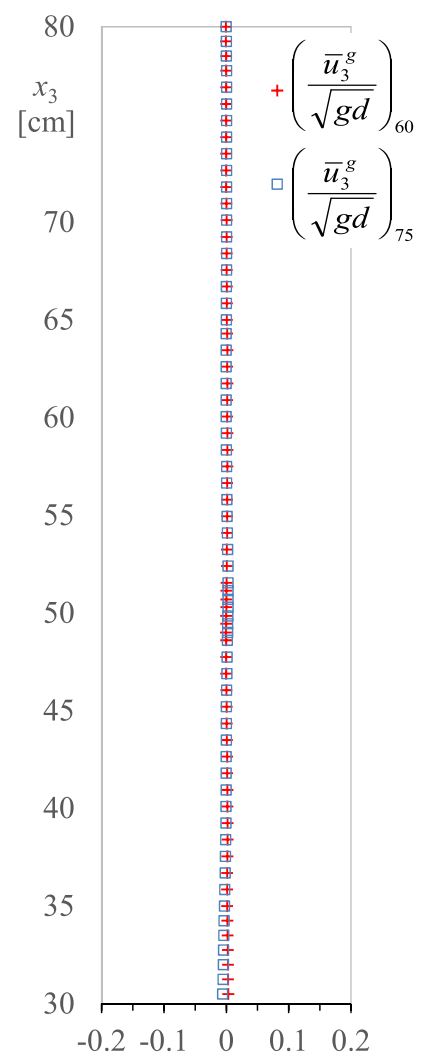

(a)

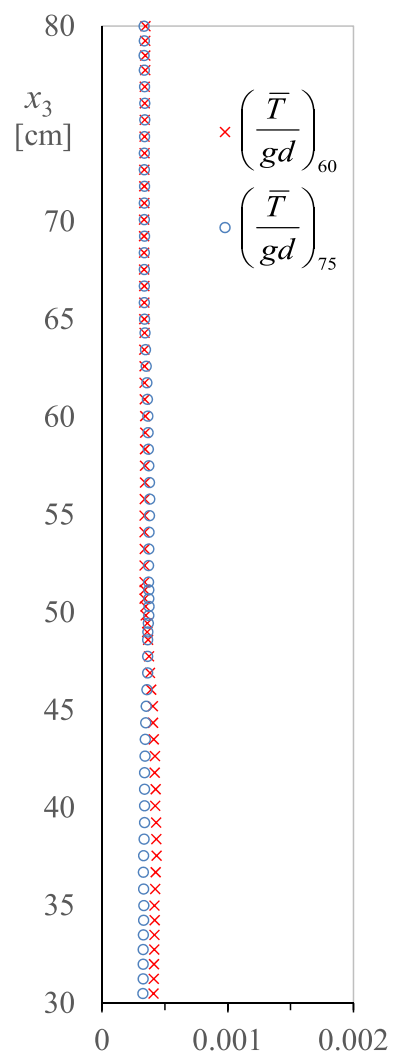

(b)

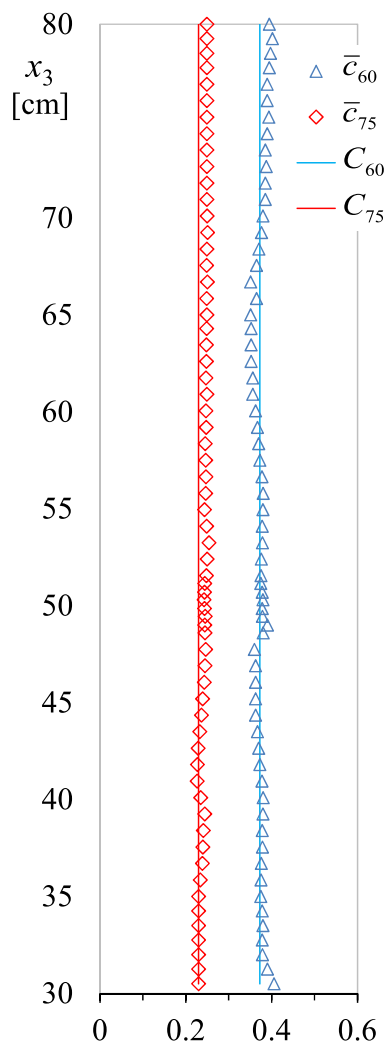

(c)

FIG. 7. Distribution along the vertical direction $x_{3}$ for two different liquid discharges: $Q=60$ and $75 \mathrm{~L} / \mathrm{min}$, respectively, of (a) the dimensionless time-averaged velocity of the granular phase, (b) the dimensionless time-averaged granular temperature; and (c) the dimensionless solid concentration (local values measured with the optical method) and the depth average concentration $C$, measured with the volumetric method.

into the average value $\overline{D_{i}}$ plus the fluctuating component $D_{i}^{\prime}$ that, by definition, is null on average.

Since the drag depends nonlinearly on the relative velocity and concentration, it is worth noting that the drag calculated with the average value of these parameters, hereafter denoted by $\overline{\overline{D_{i}}}$, is different from the averaged drag $\overline{D_{i}}$, so we can write

$$
\overline{D_{i}}=\overline{\overline{D_{i}}}+D_{R i}
$$

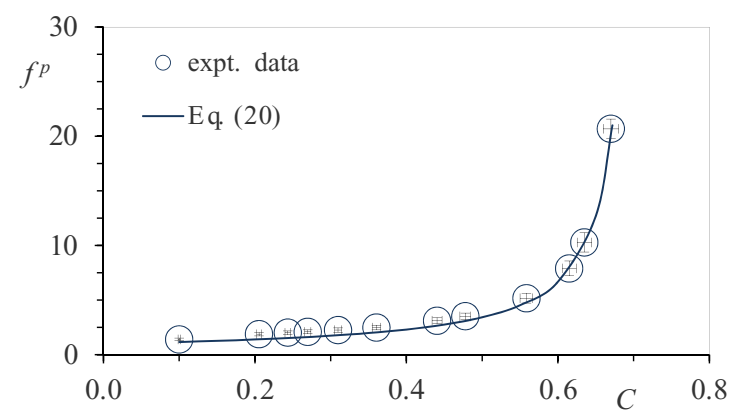

FIG. 8. Distribution of the population drag function $f^{p}$ according to Eq. (20) (solid line) and the value of the experiment (blue circles) for different values of the concentration. where the term $D_{R i}$ defined by Eq. (21) is a residual term, representing the difference between the two drag forces. Figure 9 shows a comparison between the values of the drag $\overline{D_{3}}$ calculated from the experiments with that $\overline{\overline{D_{3}}}$ calculated as a function of the averaged values of the concentration and of the velocities. Figure 9 shows also the residual drag $D_{R 3}$ calculated as the difference $\overline{D_{3}}-\overline{\overline{D_{3}}}$. At high concentrations $(C>0.3)$, the residual drag decreases upon increasing the concentration.

To derive an expression for the residual drag, we insert Eq. (6) into Eq. (7), obtaining an expression for the instantaneous drag:

$$
\begin{aligned}
& D_{i}=17.3 f^{p} \frac{\mu_{w}}{d^{2}} c\left(u_{i}^{f}-u_{i}^{g}\right)+4.5 f^{p} \frac{\sqrt{\rho_{w}} \sqrt{\mu_{w}}}{d \sqrt{d}} . \\
& c\left(u_{i}^{f}-u_{i}^{g}\right)^{1.5}+0.3 f^{p} \frac{\rho_{w}}{d} c\left(u_{i}^{f}-u_{i}^{g}\right)^{2} .
\end{aligned}
$$

We can now apply the Reynolds decomposition to the instantaneous velocities and concentration to all terms of Eq. (22), i.e., $c=\bar{c}+c^{\prime}, u_{i}^{f}=\bar{u}_{i}^{f}+u_{i}^{\prime f}$, and $u_{i}^{g}=\bar{u}_{i}^{g}+u_{i}^{g^{\prime}}$, 
and then average the equation over time, obtaining

$$
\begin{aligned}
& D_{R i \text {-corr }} \frac{d^{2}}{f^{p} \mu_{w} \bar{u}_{i}^{f}}=17.3 \overline{\frac{c^{\prime}\left(u_{i}^{\prime f}-u_{i}^{\prime g}\right)}{\bar{u}_{i}^{f}}}+\frac{27}{4} \sqrt{\frac{\overline{u_{i}^{f} d \rho_{w}}}{\mu_{w}}}\left[\left(\frac{\bar{u}_{i}^{f}-\bar{u}_{i}^{g}}{\bar{u}_{i}^{f}}\right)^{0.5 \overline{\frac{c^{\prime}\left(u_{i}^{\prime f}-u_{i}^{\prime g}\right)}{\bar{u}_{i}^{f}}}}\right] \\
& +\frac{27}{16} \sqrt{\frac{\bar{u}_{i}^{f} d \rho_{w}}{\mu_{w}}}\left[\frac{\bar{c}}{\left(\bar{u}_{i}^{f}-\bar{u}_{i}^{g}\right)^{0.5}} \frac{\overline{\left(u_{i}^{\prime}-u_{i}^{\prime g}\right)^{2}}}{\bar{u}_{i}^{f 1.5}}+\frac{1}{\left(\bar{u}_{i}^{f}-\bar{u}_{i}^{g}\right)^{0.5}} \frac{\overline{c^{\prime}\left(u_{i}^{\prime}-u_{i}^{\prime g}\right)^{2}}}{\bar{u}_{i}^{f 1.5}}\right] \\
& +0.3 \frac{\bar{u}_{i}^{f} d \rho_{w}}{\mu_{w}}\left[\bar{c} \overline{\left(\frac{u_{i}^{\prime f}-u_{i}^{\prime g}}{\bar{u}_{i}^{f}}\right)^{2}}+\frac{\overline{c^{\prime}\left(u_{i}^{\prime f}-u_{i}^{\prime g}\right)^{2}}}{\bar{u}_{i}^{f}}+2 \frac{\bar{u}_{i}^{f}-\bar{u}_{i}^{g}}{\bar{u}_{i}^{f}} \frac{\overline{c^{\prime}\left(u_{i}^{\prime}-u_{i}^{\prime g}\right)}}{\bar{u}_{i}^{f}}\right] \text {. }
\end{aligned}
$$

In deriving Eq. (23), we have approximated the term $\left(\bar{u}_{i}^{f}+u_{i}^{\prime f}-\bar{u}_{i}^{g}-u_{i}^{g}\right)^{1.5}$ with a second-order Taylor expansion as $\left(\bar{u}_{i}^{f}-\right.$ $\left.\bar{u}_{i}^{g}\right)^{1.5}+\frac{3}{2}\left(\bar{u}_{i}^{f}-\bar{u}_{i}^{g}\right)^{0.5}\left(u_{i}^{\prime f}-u_{i}^{g^{\prime}}\right)+\frac{3}{8}\left(\bar{u}_{i}^{f}-\bar{u}_{i}^{g}\right)^{-0.5}\left(u_{i}^{\prime f}-u_{i}^{g^{\prime}}\right)^{2}$.

As previously discussed in the case study, $\bar{u}_{3}^{g}=0$, so Eq. (23) reduces to

$$
\begin{aligned}
& D_{R 3-\operatorname{corr}} \frac{d^{2}}{f^{p} \mu_{w} \bar{u}_{3}^{f}}=\underbrace{17.3 \frac{\overline{c^{\prime} u_{3}^{\prime}}}{\bar{u}_{3}^{f}} \overline{c^{\prime} u_{3}^{\prime g}}}_{(i)}+\underbrace{\left.\frac{27}{4} \sqrt{\frac{\bar{u}_{3}^{f} d \rho_{w}}{\mu_{w}}}\left[\frac{\bar{c}\left(\overline{u_{3}^{\prime f}-u_{3}^{\prime g}}\right.}{\bar{u}_{3}^{f}}\right)^{2}+\left(\frac{\overline{c^{\prime} u_{3}^{\prime f}}-\overline{c^{\prime} u_{3}^{\prime g}}}{\bar{u}_{3}^{f}}\right)\right]}_{(i i)} \\
& \underbrace{+\frac{27}{16} \sqrt{\frac{\bar{u}_{3}^{f} d \rho_{w}}{\mu_{w}}}\left[\frac{\bar{c}^{\prime}\left(u_{3}^{\prime f}-u_{3}^{\prime g}\right)^{2}}{\left(\bar{u}_{3}^{f}\right)^{2}}\right]}_{(i i i)}+\underbrace{0.3 \frac{\bar{u}_{3}^{f} d \rho_{w}}{\mu_{w}}\left[\bar{c}\left(\overline{\frac{u_{3}^{\prime f}-u_{3}^{g^{\prime}}}{\bar{u}_{3}^{f}}}\right)^{2}+\left(\frac{\overline{c^{\prime} u_{3}^{\prime f}}-\overline{c^{\prime} u_{3}^{\prime g}}}{\bar{u}_{3}^{f}}\right)\right]}_{(i v)}
\end{aligned}
$$

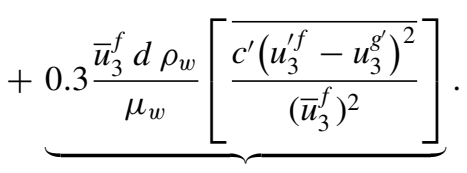

From the instantaneous and simultaneous records of granular velocity, liquid velocity, and grain concentration, we were able to obtain the different terms of Eq. (24), as reported in Table III.

The terms $i v$ and $(v)$ of Eq. (24) are proportional to the Reynolds number of the single grain, $\bar{u}_{3}^{f} d \rho_{w} / \mu_{w}$, which is much greater than 1 . For this reason, the terms (iv) and (v) are greater than the terms (ii) and (iii), which are proportional to the square root of the Reynolds number, and even greater than the term $(i)$, which does not contain this number. Furthermore, the term (iv) consists of the sum of two components: the first is the time averages of the squares of the fluctuating component of the relative velocity, i.e., the

TABLE III. Values of different terms of Eq. (24) for two values of concentrations.

\begin{tabular}{lcc}
\hline \hline$c$ & 0.22 & 0.30 \\
\hline $\bar{u}_{3}^{f} d \rho_{w} / \mu_{w}$ & 1321 & 1240 \\
(i) & -0.06 & -0.04 \\
(ii) & 4.82 & 5.07 \\
(iii) & -0.008 & -0.001 \\
(iv) & 36.78 & 34.49 \\
(v) & -0.24 & -0.38 \\
$D_{R 3-\text { corr }} d^{2} / f^{p} \bar{u}_{3}^{f} \mu_{w}$ & 41.32 & 39.13 \\
\hline \hline
\end{tabular}

sum of terms that are always positive, and the second is the correlation between the fluctuations of the concentration and of the relative velocity, which is always much smaller than the previous one.

Table III shows that (iv) is one or two orders of magnitude greater than the term $(v)$. An explanation of this difference could lie in the fact that $(v)$ represents correlations of the third order: we deduce that direct correlations between floating components of the concentration and that of the relative velocity are modest. This result is also evident by expressing the triple correlations $\overline{c^{\prime} u_{3}^{\prime} u_{3}^{\prime}}$ with a diffusive closure model, that is, assuming them as proportional to the gradient of the time-averaged concentration. As previously discussed, in this case the gradient of the time-averaged concentration is null. Finally, the residual drag term in a two-phase flow through a vertical column can be simplified as

$$
D_{R 3-\text { corr }} \simeq f^{p} \frac{\rho_{w}}{d} \bar{c} \overline{u_{3}^{\prime f} u_{3}^{\prime f}}\left(0.3+\frac{27}{16} \sqrt{\frac{\mu_{w}}{\bar{u}_{3}^{f} d \rho_{w}}}\right) .
$$

Notice that in our configuration, the simultaneous measurements of the instantaneous velocity of the liquid phase and that of the solid phase were possible only under fairly dilute conditions $(C<0.3)$, since for higher concentrations the instantaneous measure of the liquid velocity was not precise enough due to the interaction between the solid particles 


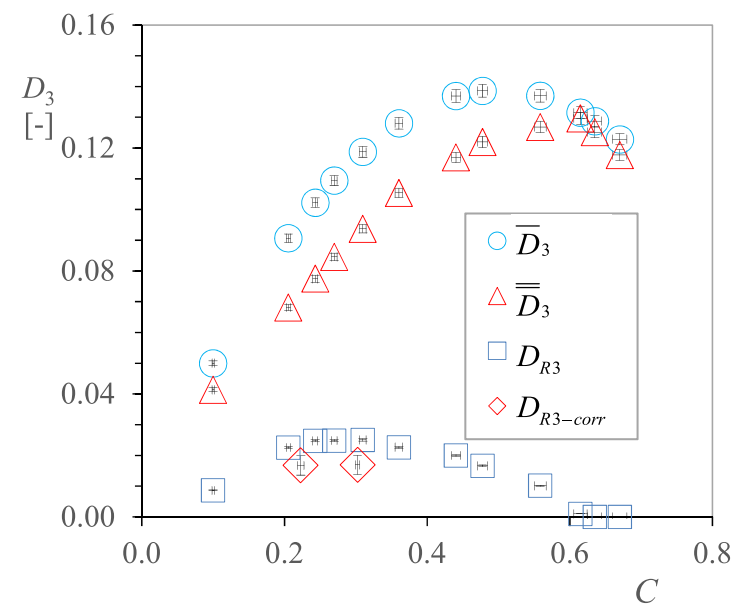

FIG. 9. Dimensionless drag forces $D_{3} /\left(g \rho_{s}\right)$, calculated according to the different definitions, as a function of the time-averaged concentration $C . \overline{D_{3}} /\left(g \rho_{s}\right)$ (blue circles) is the time-integrated value of the measured drag force and $\overline{\overline{D_{3}}} /\left(g \rho_{s}\right)$ (red triangles) is the drag calculated with the averaged values of velocities and concentration; $D_{R 3}=\left(\overline{D_{3}}-\overline{\overline{D_{3}}}\right) /\left(g \rho_{s}\right)$ (blue squares) is the residual drag calculated as the difference between the two previous definitions, and $D_{R 3 \text {-corr }} /\left(g \rho_{s}\right)$ (red rhombi) is the residual drag, approximated as in Eq. (24).

and seeding particles used to detect the water velocity. For this reason, in Fig. 9 we plot two points only (red diamonds) representing the term $D_{R 3 \text {-corr }}$. The figure shows that the assumptions made to derive the expression (24) are acceptable.

\section{CONCLUSIONS}

In the present paper, we have analyzed the problem of the resistance force between water and particles in a submerged granular flow. The experimental investigation was carried out in a vertical column with an upwind flux of water, in which we had earlier inserted heavy spherical particles.

The analysis has primarily shown the need to integrate the equations of the granular flow (equations derived from the application of the dense gas analogy) also on the time scale of the fluctuations of the water turbulence (the Reynolds scale). We arrived at this conclusion by observing that the velocity fluctuations of the solid phase are strongly correlated with the low-frequency fluctuations of the liquid phase. In addition, we compared the turbulence intensity of the fluid phase $\overline{\left(u_{i}^{\prime f} u_{i}^{\prime f}\right)}$ in the submerged granular flow with the analogous term in the absence of particles (clear water conditions). We observed that the particles do not affect substantially the turbulence intensity of the liquid phase.

At the Reynolds scale, the flow of both phases turns out to be statistically stationary and homogeneous in the main flow direction. In these conditions, we have been able to integrate the equations of momentum in the flow direction.

From the experimental analysis and from the solution of the flow equations, we derived an expression for the population drag function $f^{p}$ as the ratio between the drag force on a noncohesive population of particles and the drag force on a single isolated particle. This function depends on the average concentration of the group of particles. The experimental analysis has shown that Eq. (20) matches the experimental data for a wide range of average concentrations. This expression can be assumed to be valid in general, provided that the Basset forces and added mass are negligible.

As expected, the time-averaged drag force turns out to be different from the drag force calculated as a function of the time-averaged values of relative velocity and concentration. The residual drag, evaluated as Eq. (24), is expressed as a series of terms containing the nonlinear correlations between the fluctuating part of the velocities and the concentration. The experiments show that the second-order correlation term $\overline{\left(u_{3}^{\prime f} u_{3}^{\prime f}\right)}$ gives the most important contribution to the residual drag in the column flow. This term represents the turbulence intensity of the fluid phase. This result is not surprising since the longitudinal velocity gradient of the granular phase is zero.

\section{ACKNOWLEDGMENTS}

This work was carried out in the frame of the collaborative international consortium, STEEP STREAMS, under the ERANET Cofund WaterWorks 2014 Call. This ERA-NET is an integral part of the 2015 Joint Activities developed by the Water Challenges for a Changing World Joint Programme Initiative (Water JPI). This research was also funded by Fondazione Caritro (Grant No. 2014.0374). The authors are grateful to the two anonymous reviewers for thoughtful reviews that helped improve the manuscript.
[1] H. M. Jaeger, S. R. Nagel, and R. P. Behringer, Rev. Mod. Phys. 68, 1259 (1996).

[2] C. S. Campbell, Annu. Rev. Fluid Mech. 22, 57 (1990).

[3] I. Goldhirsch, Annu. Rev. Fluid Mech. 35, 267 (2003).

[4] S. Herminghaus, Adv. Phys. 54, 221 (2005).

[5] A. Armanini, M. Larcher, E. Nucci, and M. Dumbser, Adv. Water Res. 63, 1 (2014).

[6] A. Armanini, J. Hydraul. Res. 51, 111 (2013).

[7] T. Takahashi, Debris Flow (Balkema, Rotterdam, 1991).

[8] A. Armanini, L. Fraccarollo, and G. Rosatti, Comput. Geosci. 35, 993 (2009).

[9] R. M. Iverson, Rev. Geophys. 35, 245 (1997).
[10] A. Armanini, H. Capart, L. Fraccarollo, and M. Larcher, J. Fluid Mech. 532, 269 (2005).

[11] E. B. Pitman and L. Le, Philos. Trans. Ser. A 363, 1573 (2005).

[12] S. P. Pudasaini, J. Geophys. Res.: Earth Surf. 117, F03010 (2012).

[13] C. Truesdell, The Elements of Continuum Mechanics (Springer, New York, 1984).

[14] C. Ancey and P. Evesque, Phys. Rev. E 62, 8349 (2000).

[15] F. da Cruz, S. Emam, M. Prochnow, J.-N. Roux, and F. Chevoir, Phys. Rev. E 72, 021309 (2005).

[16] J. T. Jenkins and S. B. Savage, J. Fluid Mech. 130, 187 (1983).

[17] I. Goldhirsch, Powder Technol. 182, 130 (2008). 
[18] S. B. Savage, Adv. Appl. Mech. 24, 289 (1984).

[19] J. T. Jenkins, Granular Matter 10, 47 (2007).

[20] A. Armanini, M. Larcher, and L. Fraccarollo, Phys. Rev. E 79, 051306 (2009).

[21] G. Lois, A. Lemaître, and J. M. Carlson, Phys. Rev. E 72, 051303 (2005).

[22] C. Lun and S. Savage, Acta Mech. 63, 15 (1986).

[23] J. Chauchat and S. Guillou, J. Geophys. Res.: Oceans 113, C11017 (2008).

[24] C. Meruane, A. Tamburrino, O. Roche et al., J. Fluid Mech. 648, 381 (2010).

[25] L. Mazzei and P. Lettieri, Chem. Eng. Sci. 62, 6129 (2007).

[26] J. M. Coulson, J. F. Richardson, and J. R. Backhurst, Chemical Engineering: Particle Technology and Separation Processes, Vol. 2 (Butterworth-Heinemann, 1990).

[27] J. Dallavalle, Micrometrics (Pitman, London, 1948).

[28] L.C.v. Rijn, J. Hydraul. Eng. 110, 1613 (1984).

[29] J. Richardson and W. Zaki, Chem. Eng. Sci. 3, 65 (1954).

[30] Y. Makkawi and P. Wright, Chem. Eng. Sci. 58, 2035 (2003).

[31] R. Di Felice, Int. J. Multiphase Flow 20, 153 (1994).

[32] H. Darcy, The Public Fountains of the City of Dijon (Hunt Publishing Company, Dubuque, 1856), English Translation by Patricia Bobeck.
[33] J. Bear, Dynamics of Fluids in Porous Media (Elsevier, New York, 1972).

[34] M. A. van der Hoef, R. Beetstra, and J. A. M. Kuipers, J. Fluid Mech. 528, 233 (2005).

[35] P. Carman, Flow of Gases through Porous Media (Butterworths, London, 1956).

[36] H. Watanabe, Powder Technol. 19, 217 (1978).

[37] J. M. Ham, S. Thomas, E. Guazzelli, G. M. Homsy, and M. Anselmet, Intl J. Multiphase Flow 16, 171 (1990).

[38] M. Nicolas, J. Chomaz, D. Vallet, and E. Guazzelli, Phys. Fluids 8, 1987 (1996).

[39] P. Duru, M. Nicolas, J. Hinch, and E. Guazzelli, J. Fluid Mech. 452, 371 (2002).

[40] H. Capart, D. Young, and Y. Zech, Exp. Fluids 32, 121 (2002).

[41] R. A. Bagnold, Proc. R. Soc. London, Ser. A 225, 49 (1954).

[42] T.-J. Hsu, J. T. Jenkins, and P. L.-F. Liu, J. Geophys. Res.: Oceans (1978-2012) 108, 2223 (2003).

[43] T.-J. Hsu, J. T. Jenkins, and P. L.-F. Liu, Proc. R. Soc. London, Ser. A 460, 2223 (2004).

[44] B. P. Greimann, M. Muste, and F. M. Holly Jr., J. Hydraul. Res. 37, 479 (1999). 